\title{
Comparison of the B field dependency of plasma parameters of a weakly magnetized inductive and Helicon hydrogen discharge
}

\author{
S Briefi ${ }^{1}$, P Gutmann ${ }^{1}$, D Rauner ${ }^{1,2}$ and U Fantz ${ }^{1,2}$ \\ ${ }^{1}$ AG Experimentelle Plasmaphysik, Universität Augsburg, 86135 Augsburg, \\ Germany \\ ${ }^{2}$ Max-Planck-Institut für Plasmaphysik, Boltzmannstrasse 2, 85748 Garching, \\ Germany \\ E-mail: stefan.briefi@physik.uni-augsburg.de
}

\begin{abstract}
The discharge properties of a weakly magnetized inductively coupled hydrogen discharge (operating pressure $1 \mathrm{~Pa}$ ) are evaluated by using optical emission spectroscopy. The behaviour of the electron density $\mathrm{n}_{e}$, temperature $\mathrm{T}_{e}$ and the density ratio of atomic to molecular hydrogen $n_{\mathrm{H}} / \mathrm{n}_{\mathrm{H}_{2}}$ with varying magnetic field strength (up to $12 \mathrm{mT}$ ) is investigated. The results obtained from the OES measurements performed with a line of sight directed along the central axis of the cylindrical discharge vessel are compared to the case when the ICP antenna is replaced by a Nagoya-type-III Helicon antenna. In the ICP case, the electron temperature and density at the axis of the cylindrical discharge vessel decrease with increasing magnetic field due to the hindered radial electron diffusion. This results in a gradual transition from a homogeneous radial emission profile to a hollow profile with minimal emission in the discharge centre. Concerning the density ratio of atomic to molecular hydrogen, one obtains very high values of up to 0.32 at low B field and a decreasing behaviour with higher magnetic fields. For the Helicon case, the obtained values of $n_{e}$ and $\mathrm{T}_{e}$ are virtually unaffected by the external magnetic field. Furthermore, a hollow radial emission profile is observed already at low $\mathrm{B}$ field strengths. In the Helicon setup one obtains an increasing trend for $n_{\mathrm{H}} / \mathrm{n}_{\mathrm{H}_{2}}$ with a maximum of about 0.2 at $12 \mathrm{mT}$.
\end{abstract}

PACS numbers: $52.70 . \mathrm{Kz}, 52.25 . \mathrm{Xz}, 52.50$.Qt, $52.80 . \mathrm{Pi}$

Submitted to: Plasma Sources Sci. Technol.

Keywords: Optical emission spectroscopy, Inductively coupled plasma, Helicon discharge, Hydrogen discharge

\section{Introduction}

Discharge generation via inductive coupling is one of the most widespread implementations concerning low pressure plasma sources for example in the 
semiconductor manufacturing industry $[1,2]$. This arises from the fact that the electron density is much higher and the sheath potential and therefore the ion energy is much lower in an ICP than in a capacitively coupled discharge. Within the last years considerable experimental and theoretical research has been carried out concerning the effect of a weak external DC magnetic field on the discharge parameters of an ICP (see for example [3-7]). The aim was to enhance the RF coupling efficiency and to improve the stability of low-pressure operation. The investigations were primarily performed in argon at pressures between 0.2 and $10 \mathrm{~Pa}$, with B field strengths of up to $10 \mathrm{mT}$ and $\mathrm{RF}$ powers of a few hundred watts or lower.

Another discharge generation method is Helicon coupling. It relies on wave heating mechanisms in magnetized cylindrical discharges and is well-known for producing plasmas with a high degree of ionization very efficiently [8-10]. The high-density Helicon mode is typically achieved for powers in the $\mathrm{kW}$-range applying external magnetic fields around a few hundred mT by using argon at a pressure below $1 \mathrm{~Pa}$ as working gas. However, Helicon discharges can also be operated with lower RF powers and B fields - in the range of a few hundred watts and a few $\mathrm{mT}$. The RF coupling relies in this operational mode on a mixture of inductive and Helicon coupling. Also a prominent feature of Helicon discharges occurs at low magnetic field strength: the socalled low field peak which is a local enhancement of the RF coupling efficiency [11]. The specific physical reason behind this peak is not well understood yet (some explanatory approaches are given in $[12,13])$.

In this paper a comparison between a weakly magnetized ICP and a Helicon discharge is performed. The external magnetic fields is varied between 0 and $12 \mathrm{mT}$. The investigations were carried out in cw operation (Helicon discharges are very often operated in pulsed mode to lower the heat loads) at an experimental setup that utilizes a cylindrical discharge vessel (diameter $10 \mathrm{~cm}$, length $40 \mathrm{~cm}$ ). This design is somewhat different compared to the typical plasma chambers used for material processing but the geometry is required as boundary condition to allow the propagation of Helicon waves. Furthermore, the aim is the identification of fundamental differences of the two coupling methods rather than realizing Helicon coupling for a processing chamber (which has already been done by placing a cylindrical discharge vessel on top of the reaction chamber, see for example [14]). As for material treatment purposes hydrogen plasmas play an important role, the investigations are carried out using hydrogen as working gas. Focus is put on the behaviour of the electron temperature $\mathrm{T}_{e}$ and density $\mathrm{n}_{e}$ with respect to the external magnetic field strength. As atomic hydrogen - which is created via dissociation processes - serves as reactive or etching species in some applications, also the density ratio of atomic to molecular hydrogen $n_{\mathrm{H}} / \mathrm{n}_{\mathrm{H}_{2}}$ is considered.

\section{Experimental setup and diagnostic methods}

A sketch of the experimental setup is shown in figure 1. The discharge vessel is made out of quartz glass (length $40 \mathrm{~cm}$, diameter $10 \mathrm{~cm}$ ) and is attached to the vacuum system at 
both ends of the cylinder. As RF antenna either a conventional helical ICP antenna (5 windings) or a Nagoya-type-III Helicon antenna (coupling to the $|m|=1$ Helicon modes [15]) is applied. In any case, the antenna is made out of copper tubes (6 mm diameter) and it is connected to the matching network via Swagelok tube fittings (the antenna legs have a length of about $20 \mathrm{~cm}$ ). The utilized RF generator operates at a frequency of $13.56 \mathrm{MHz}$ and has a maximum power of $600 \mathrm{~W}$. The external magnetic field, which is directed parallel to the cylindrical axis of the discharge vessel, is generated by a pair of Helmholtz coils (380 windings and $18 \mathrm{~cm}$ radius each) and reaches a maximum strength of about $12 \mathrm{mT}$. All investigations presented in this paper are carried out at the maximum available RF power of $600 \mathrm{~W}$ (reflected power $0 \mathrm{~W}$ ) and at a filling pressure of $1 \mathrm{~Pa}$ in hydrogen (measured gas-type independently via a temperaturestabilized capacitive pressure gauge before plasma ignition, typical error $\pm 0.15 \%$ of the pressure reading). The base pressure which is measured via a cold-cathode pressure gauge is around $5 \times 10^{-7}$ mbar. Both pressure gauges are attached at the left side of the discharge vessel. The working gas is fed to the discharge vessel at a constant flow rate of $5 \mathrm{sccm}$ (by using the mass flow meter EL-FLOW from Bronkhorst, typical error $\pm 2.5 \%$ of the flow setting) from the left and is pumped at the right side where the pressure is adjusted via limiting the pumping speed.

\section{ICP configuration}

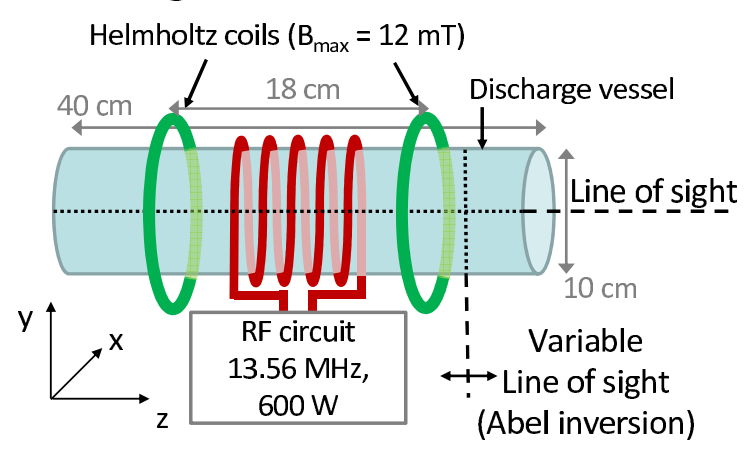

\section{Helicon configuration}

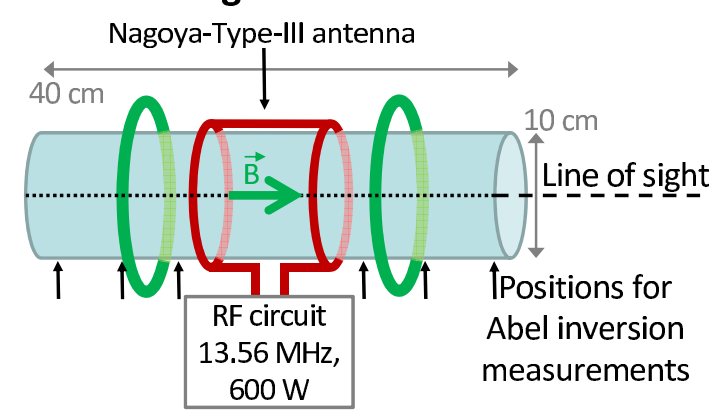

Figure 1. Sketch of the experimental setup. In the upper part, the ICP configuration with the helical RF antenna is shown whereas in the bottom part the Helicon configuration with the Nagoya-type-III antenna is depicted. The short black arrows in the bottom part indicate the different z-positions where Abel inversion measurements were carried out. 
As diagnostic method optical emission spectroscopy (OES) has been used. The measurements were carried out with an absolutely calibrated high-resolution spectrometer $\left(\Delta \lambda_{\mathrm{FWHM}} \approx 50 \mathrm{pm}\right)$ along an axial line of sight (radially centred). In order to obtain plasma parameters from the OES measurements, the collisional radiative models Yacora $\mathrm{H}$ for atomic [16] and Yacora $\mathrm{H}_{2}$ for molecular hydrogen $[17,18]$ are applied. As input parameters for the models the pressure (fixed at $1 \mathrm{~Pa}$ as already stated) and the gas temperature are required. The latter has been determined from the molecular Fulcher transition $\left(d^{3} \Pi_{u} \rightarrow a^{3} \Sigma_{g}^{+}\right.$, located between 590 and $\left.650 \mathrm{~nm}\right)$ according to $[19,20]$. In order to evaluate plasma parameters from OES, the absolute intensities of the atomic Balmer lines $\mathrm{H}_{\alpha}$ to $\mathrm{H}_{\delta}$ and of the integrated emission of the Fulcher transition are considered. The intensities calculated by the models have been fitted to the measurements by using the electron temperature, the electron density and the density ratio of atomic to molecular hydrogen $n_{\mathrm{H}} / \mathrm{n}_{\mathrm{H}_{2}}$ as fitting parameters. Since the intensities measured by OES represent line-of-sight averaged values, also the obtained values of $\mathrm{T}_{e}, \mathrm{n}_{e}$ and $n_{\mathrm{H}} / \mathrm{n}_{\mathrm{H}_{2}}$ have to be treated as line-of-sight averaged.

It is expected that the external magnetic field influences the radial distribution of the plasma parameters due to its influence on the radial diffusion of charged particles. Therefore, radial profiles of the $\mathrm{H}_{\beta}$ emission have been determined by means of Abel inversion. Assuming a cylindrically symmetric emission, the radial profile can be calculated from lateral OES measurements by solving the Abel equation numerically (here the Hankel-Fourier solving method has been applied [21]). Detailed investigations showed that lateral measurements at 26 equidistant positions, ranging from the lower edge of the discharge tube to the upper edge, lead to a reliable reconstruction of the radial emission profile. Measurements of the radial emission profiles were always performed at six different z-positions for each $\mathrm{B}$ field value: close to the end of the discharge vessel, between the end and the Helmholtz coil and between the coil and the RF antenna, each on the left and on the right hand side (indicated by the small black arrows in the bottom part of figure 1) in order to resolve a possible variation of the emission profile shape in z-direction. As the OES measurements for the Abel inversion take several minutes to record, it has been assured that the discharge reached a stable equilibrium. This is typically the case after 30 minutes of operation. In order to assure the temporal stability of the plasma, its emission is monitored by a low-resolution spectrometer at the axial line of sight with a time resolution of 1 second (averaging over 5 recordings). When OES measurements are performed, the temporal variation of the discharge emission is below $1 \%$.

For Abel inversion it is generally the case that the error of the reconstructed radial profile increases with decreasing radius $r$. This is because the calculation starts at the outermost shell and then goes step by step inwards towards $r=0$ (an example of a quantitative consideration of this error can be found in [22] for example). Therefore, the obtained radial emission profile can be considered reliable with respect to the absolute value close to the plasma edge but an error of several $10 \%$ can occur towards the discharge centre. Due to this fact, only the general shape of the radial emission profiles 
is compared for different experimental conditions in the following.

\section{Results and discussion}

\subsection{ICP configuration}

In the left part of figure 2 the measured emissivity of the $\mathrm{H}_{\alpha}$ line and of the full Fulcher transition is shown for a variation of the external magnetic field. In general, the relative trends of the other Balmer lines are the same as for the $\mathrm{H}_{\alpha}$ line therefore their emissivity is not depicted in figure 2. When the B field is switched on, a jump-like increase in both the atomic and molecular emission is observable. This jump can be attributed to a change in the plasma distribution along the line of sight. When no external magnetic field is present, the discharge is concentrated under the RF antenna. Only faint emission is visible towards the ends of the discharge vessel. Applying a magnetic field leads to a uniform plasma distribution throughout the whole discharge vessel. This effect can be explained by the guiding influence of the magnetic field lines on the charged particles. However, the plasma fills out the whole discharge volume with and without B field. Therefore, a constant length of $40 \mathrm{~cm}$ for the line of sight has always been used for the calculation of the emissivity. This means the change in the plasma distribution along the line of sight is not considered in the data evaluation. A further increase of the external magnetic field leads to a steady decrease of the emissivity. The evaluation of the measured absolute intensities with the collisional radiative models yields also a decrease of $\mathrm{T}_{e}$ from $4.3 \pm 0.22$ to $2.9 \pm 0.15 \mathrm{eV}$ and of $\mathrm{n}_{e}$ from $1 \times 10^{17} \pm 0.2 \times 10^{17}$ to $5 \times 10^{16} \pm 1 \times 10^{16} \mathrm{~m}^{-3}$, shown in the right part of figure 2 .
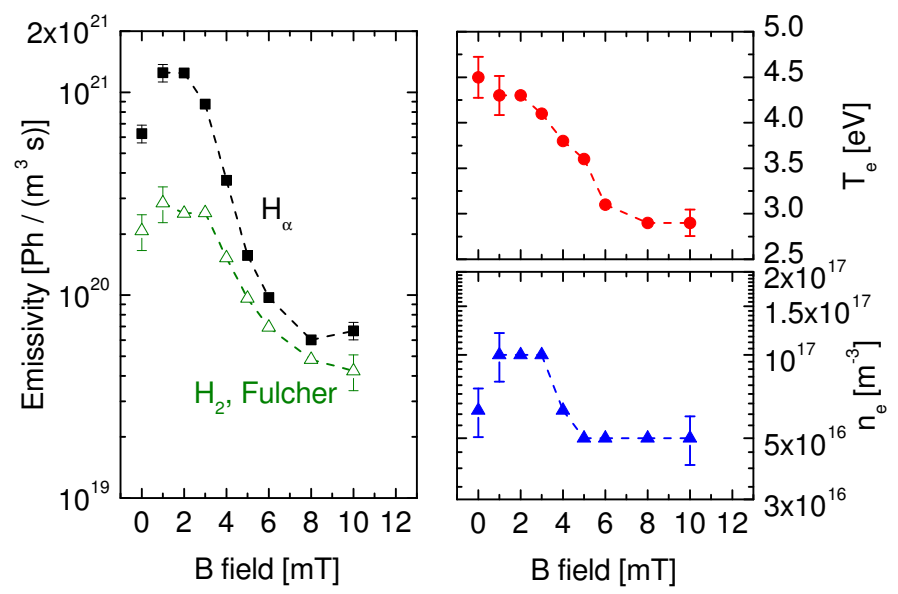

Figure 2. Emissivity of the atomic $\mathrm{H}_{\alpha}$ and the molecular Fulcher radiation at varying external magnetic field for ICP configuration (left part) together with the electron temperature and density evaluated from the OES measurements (right part) performed along the axial (radially centered) line of sight.

Figure 3 shows the density ratio of atomic to molecular hydrogen obtained from the OES measurements for varying magnetic fields. The density ratio decreases from 
around 0.3 at low B fields to around 0.1 at high fields which reflects the trends of $\mathrm{T}_{e}$ and $\mathrm{n}_{e}$. The absolute values of $n_{\mathrm{H}} / \mathrm{n}_{\mathrm{H}_{2}}$ are remarkably high as typically only values around 0.05 or below are obtained for low pressure low temperature discharges in hydrogen at these RF power levels.

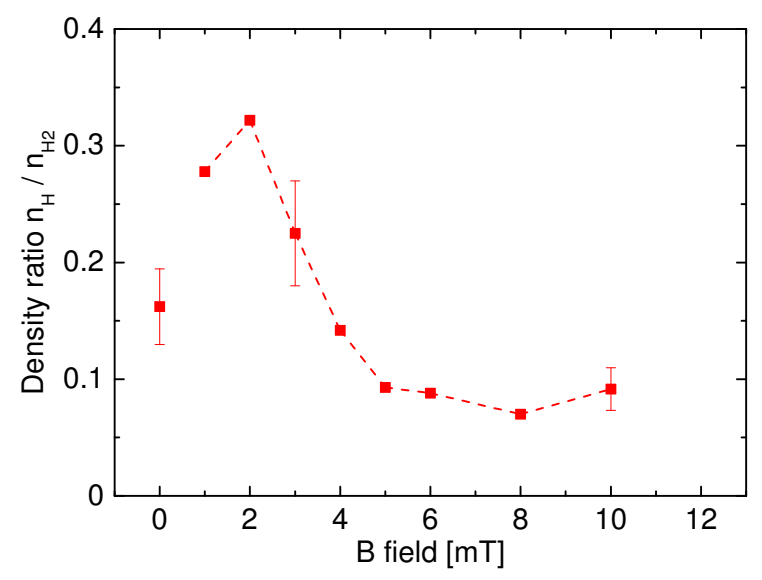

Figure 3. Density ratio of atomic to molecular hydrogen at varying external magnetic field determined from OES measurements performed along the axial (radially centered) line of sight for the ICP configuration.

Also the radial intensity profiles, determined via the Abel inversion technique, are influenced by the external magnetic field. Figure 4 shows the radial emission profiles of $\mathrm{H}_{\beta}$ obtained for magnetic field strengths of 0,3 and $10 \mathrm{mT}$. The radial emission profiles did not show a distinct dependence on the z-position. Therefore the position between the Helmholtz coil and the RF antenna on the right hand side has been chosen for figure 4 exemplarily. Without external magnetic field and for $B=3 \mathrm{mT}$, the profile is quite flat in the middle of the vessel and shows a steep decrease towards the discharge vessel wall. The fluctuations in the flat region are not considered real, see the discussion on the reliability of profiles determined via Abel inversion in section 2. For increasing magnetic field strengths, the profile changes gradually towards a deep hollow profile with a minimum in the discharge centre (shown for $B=10 \mathrm{mT}$ ).

In order to understand the mechanisms leading to the change in the radial emission profiles with increasing magnetic field, one has to look at those plasma processes that lead to Balmer line emission for the present discharge parameters. A thorough analysis of the collisional radiative models yields that the exited states in the $\mathrm{H}$ atom are primarily populated via electron impact excitation of ground state hydrogen atoms and dissociative excitation of $\mathrm{H}_{2}$. All other processes like dissociative recombination of $\mathrm{H}_{2}^{+}$ or mutual neutralisation with $\mathrm{H}^{-}$can be neglected as their summarized contribution to the total excitation of the upper states of the Balmer series is around $10 \%$. Even dissociative excitation of $\mathrm{H}_{2}$ only contributes slightly due to the high density ratio of atomic to molecular hydrogen (see figure 3). The emissivity $\epsilon_{i k}$ of the Balmer lines can 


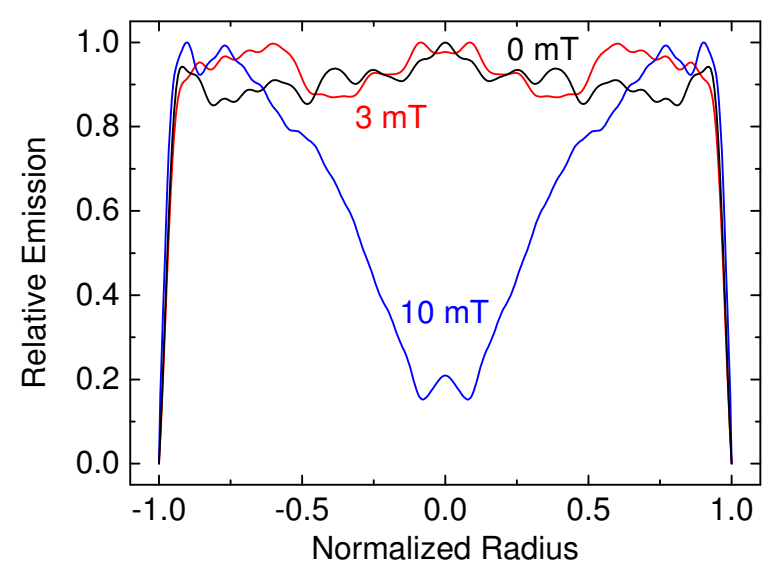

Figure 4. Radial emission profiles of $\mathrm{H}_{\beta}$ determined via Abel inversion for the ICP configuration at $B=0,3$ and $10 \mathrm{mT}$. The profiles have been measured between the coil and the RF antenna on the right hand side of the antenna.

then be expressed by [16]

$$
\epsilon_{i k}=n_{i} A_{i k}=n_{\mathrm{H}} n_{e} R_{H, i}\left(T_{e}, n_{e}, n_{H}\right) .
$$

In the equation, $R_{H, i}$ denotes the population coefficient of the excited state $i$ coupled to the atomic hydrogen density, $\mathrm{n}_{i}$ is the density of the excited state and $A_{i k}$ the transition probability for spontaneous emission from state $i$ to $k$ with $k<i$. As the lifetime of the exited states in the $\mathrm{H}$ atom is very short (in the range of $10 \mathrm{~ns}$, their motion through the vessel happens in the range of several $\mu \mathrm{s}$ ) an immediate optical decay can be assumed after the excitation. Therefore, the radial intensity profile has to arise from a radial profile of $\mathrm{n}_{\mathrm{H}}, \mathrm{n}_{e}$ or $\mathrm{T}_{e}$.

With increasing external magnetic field strength, the radial diffusion of charged particles is more and more hindered. The diffusion of hot electrons from the region close to the discharge vessel wall (where RF heating primarily occurs as the skin depth is below $2 \mathrm{~cm}$ for the investigated discharges) towards the discharge centre is hence reduced. This leads to a drop for both $\mathrm{n}_{e}$ and $\mathrm{T}_{e}$ at the axis and to a hollow radial profile. Such an effect of an external B field is very well known in literature and described as transition from non-local to local electron kinetics (see for example [3, 6, 23] for experimental and [24] and references therein for modelling investigations). Therefore the gradually changing electron kinetics can explain the observed gradual change of the radial intensity profile. Also the decrease of the electron temperature and density with increasing B field (see figure 2) can be explained as the OES measurements have been performed at a line of sight directed along the central axis of the discharge.

\subsection{Helicon configuration}

The left part of figure 5 shows the emissivity of the $\mathrm{H}_{\alpha}$ and the Fulcher transition obtained at varying magnetic field strengths. It should be noted that the generation of Helicon waves is not possible without external magnetic field. Therefore, the RF 
coupling is solely inductive for the Helicon setup at $B=0 \mathrm{mT}$. Compared to the ICP configuration, the atomic emission is the same, but the molecular emission is slightly higher without magnetic field. Switching on the B field again leads to a jump in the detected emission. However, in the Helicon mode the jump is directed to lower emissivities whereas it went to higher values in ICP mode. In addition, the behaviour of the plasma emission with increasing B field is different in Helicon configuration compared to ICP: the typical low-field peak can be observed, followed by an increasing atomic emission and a virtually constant molecular emission at higher field strengths.
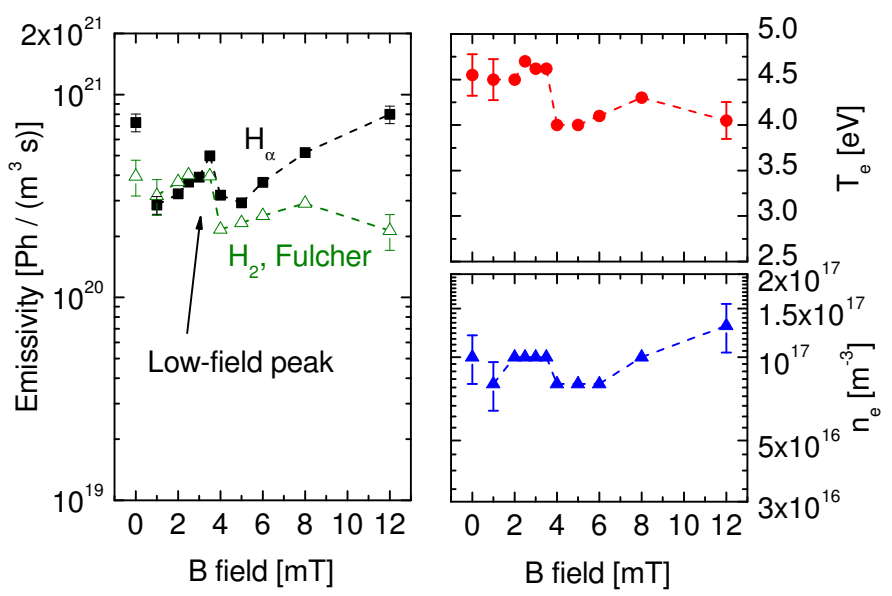

Figure 5. Emissivity of the atomic $\mathrm{H}_{\alpha}$ and the molecular Fulcher radiation at varying external magnetic field for Helicon configuration (left part) together with $\mathrm{T}_{e}$ and $\mathrm{n}_{e}$ evaluated from the OES measurements (right part) performed along the axial (radially centered) line of sight.

Concerning the electron temperature and density (right part of figure 5), the variation of the magnetic field has very little influence. $\mathrm{T}_{e}$ only drops from about 4.5 to about $4.0 \mathrm{eV}$ at high magnetic fields. The electron density stays virtually constant around $1 \times 10^{17} \mathrm{~m}^{-3}$ with a slight increase in the region of the low-field peak and at high B field. This general behaviour is typical for Helicon discharges at magnetic field strengths in the range of a few $\mathrm{mT}$ [11] and explains the observed discharge emission trends. As expected, a transition of the discharge into the high-density full Helicon mode, which is typically accompanied by a large jump in the electron density, is not achieved due to the limited RF power and magnetic field strengths.

In figure 6 the density ratio of atomic to molecular hydrogen is shown for varying magnetic fields. One obtains a steady increase of the density ratio from 0.06 to 0.2 with a slight peak in the region of the low-field peak. This reflects the behaviour of the electron temperature and density similarly as for the ICP configuration. Considering the absolute values, the density ratio is up to a factor of six smaller in the Helicon mode compared to the ICP setup below $6 \mathrm{mT}$ of magnetic field. However, at high B field values, the density ratio is higher in the Helicon configuration.

For the Helicon antenna, the applied external magnetic field allows additional discharge heating via Helicon and Trivelpiece-Gould waves. This heating power is 


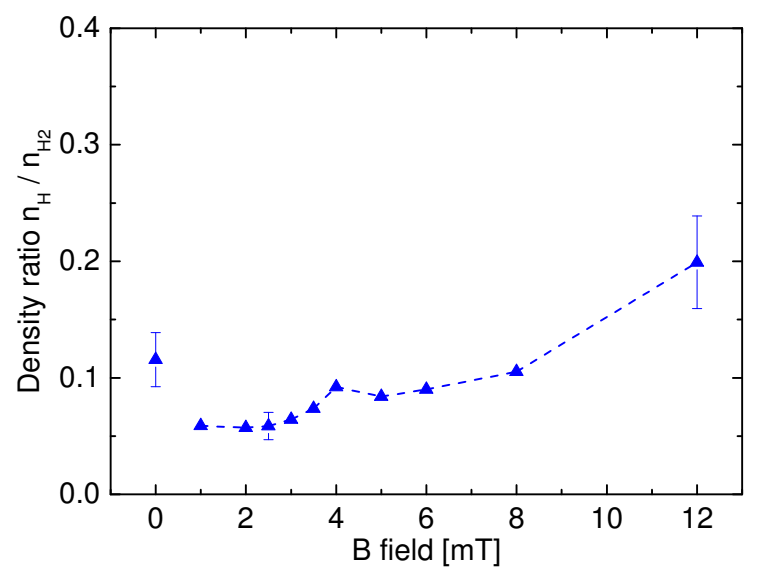

Figure 6. Density ratio of atomic to molecular hydrogen at varying external magnetic field determined from OES measurements performed along the axial (radially centered) line of sight for the Helicon configuration.

primarily deposited close to the vessel wall [8] similarly to conventional RF heating. Hence, no large difference to the ICP case is expected concerning the radial intensity profile variation with increasing magnetic field. But as shown in figure 7 , a hollow radial emission profile is already obtained at very low B fields. This explains the observed jump-like decrease in the plasma emission when switching on the B field (see figure 5): compared to a flat profile, a hollow profile leads to a strong decrease of the plasma emission in the discharge centre where the axial line of sight is located and hence to a smaller measured $\mathrm{H}_{\alpha}$ and Fulcher emissivity. However, at such low field strengths the charged particle diffusion is barely hindered (also the electron temperature and density determined from the OES measurements are not reduced, see figure 5) and can therefore not be the reason behind the observed hollow profile.

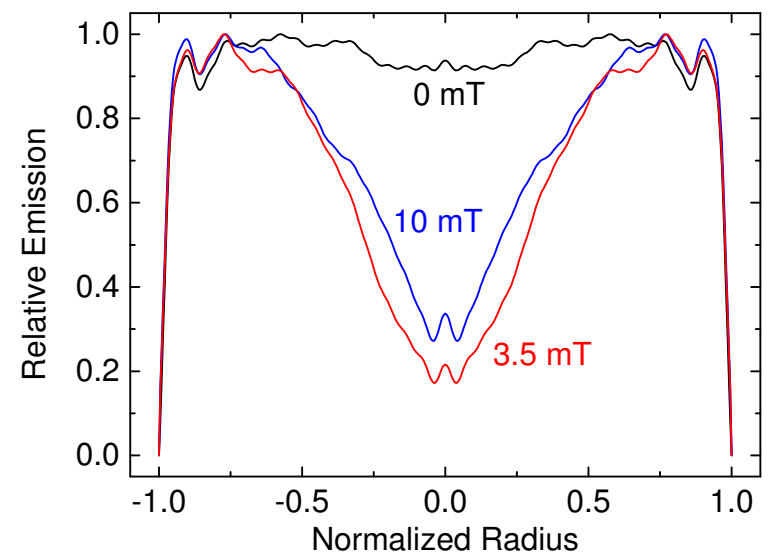

Figure 7. Radial emission profiles of $\mathrm{H}_{\beta}$ determined via Abel inversion for the Helicon configuration at $B=0,3.5$ (where the maximum of the low-field peak is located) and $10 \mathrm{mT}$. The profiles have been measured between the coil and the RF antenna on the right hand side. 
As stated in section 3.1, the Balmer emission basically depends on $\mathrm{T}_{e}, \mathrm{n}_{e}$ and the atomic hydrogen density. Therefore, besides the electron temperature and density, a hollow emission profile can also be caused by a hollow radial profile of $n_{H}$. Such an effect where the density of neutrals in the centre of a discharge is strongly reduced is called neutral depletion. In general, this can happen due to a radial profile in the gas temperature $\mathrm{T}_{\text {gas }}$ or due to a high electron pressure, which has to be considered in the total pressure balance (see [25] for example for a detailed explanation of the different processes). A radial profile of the gas temperature has been determined by evaluating $\mathrm{T}_{\text {gas }}$ from the Fulcher emission at each lateral position and a subsequent Abel inversion. However, only a slightly peaked profile ranging from $700 \mathrm{~K}$ at the wall to $740 \mathrm{~K}$ in the centre of the discharge vessel has been obtained (typical error $\pm 50 \mathrm{~K}$ ). Furthermore, the electron pressure also stays below $1 \%$ of the filling pressure in any case. Therefore the occurrence of neutral depletion and hence of a hollow radial profile of $n_{H}$ can basically be ruled out.

One should be aware that a hollow radial profile in the electron temperature and density can still be present. It just cannot be caused by the hindered charged particle diffusion. Furthermore, no drop is observed for $\mathrm{T}_{e}$ and $\mathrm{n}_{e}$, when the B field is switched on. If a hollow profile of those parameters is present, it must arise from a strong increase of $\mathrm{T}_{e}$ and $\mathrm{n}_{e}$ close to the vessel wall and no decrease of the values in the discharge centre. In order to gain more insight into the processes behind the hollow emission profile formation at low magnetic field strengths in the Helicon case, spatially resolved Langmuir probe measurements are planned in the future.

\section{Summary}

Two different RF coupling concepts, which are well-known for generating discharges with high efficiency, have been investigated especially concerning their application to hydrogen plasmas: a conventional ICP enhanced with a weak DC magnetic field and lowfield Helicon coupling. In the ICP configuration of the experimental setup, it has been shown that a low magnetic field in the range between 1 and $3 \mathrm{mT}$ enhances the coupling efficiency. A higher field leads to a decrease of the measured electron temperature, density and density ratio of atomic to molecular hydrogen due to the hindered radial diffusion of charged particles, which leads to a hollow radial discharge emission profile. In the Helicon configuration a different behaviour has been observed: a hollow profile is already obtained at low external magnetic fields, the reason for it is not clear yet. In contrast to the behaviour observed in ICP setup, the values of $\mathrm{T}_{e}, \mathrm{n}_{e}$ and $n_{\mathrm{H}} / \mathrm{n}_{\mathrm{H}_{2}}$ increase with increasing $\mathrm{B}$ field. In order to gain more insight in these trends and in the constitution of the radial emission profiles, spatially resolved Langmuir probe measurements are foreseen. 


\section{Acknowledgments}

The authors would like to thank the Deutsche Forschungsgemeinschaft (DFG) for their support within the project BR 4904/1-1.

\section{References}

[1] Hopwood J 1992 Plasma Sources Sci. Technol. 1109

[2] Lieberman M A and Lichtenberg A J 2005 Principles of Plasma Discharges and Materials Processing (John Wiley \& Sons, New York)

[3] Lee H J, Yang I D and Whang K W 1996 Plasma Sources Sci. Technol. 5383

[4] Chung C, Kim S S and Chang H Y 2002 Phys. Rev. Lett. 88(9) 095002

[5] Godyak V A and Alexandrovich B M 2004 Phys. Plasmas 11 3553-3560

[6] Lee, M and Chung, C 2007 Phys. Plasmas 14103507

[7] Kim Y D, Lee Y K, Lee H C and Chung C W 2013 Phys. Plasmas 20023505

[8] Chen F F 2015 Plasma Sources Sci. Technol. 24014001

[9] Boswell R W and Chen F F 1997 IEEE Transactions on Plasma Science 251229 1244

[10] Chen F F and Boswell R W 1997 IEEE Transactions on Plasma Science 25 12451257

[11] Chen F F, Jiang X, Evans J D, Tynan G and Arnush D 1997 Plasma Phys. Control. Fusion 39 A411-A420

[12] Cho S 2006 Phys. Plasmas 13033504

[13] Chen F F 2003 Phys. Plasmas 10 2586-2592

[14] Perry A J and Boswell R W 1989 Appl. Phys. Lett. 55 148-150

[15] Chen F F 1996 Phys. Plasmas 3 1783-1793

[16] Wünderlich D, Dietrich S and Fantz U 2009 J. Quant. Spectrosc. Radiat. Transfer $11062-71$

[17] Wünderlich D 2004 Ph.D. thesis, University of Augsburg

[18] Wünderlich D and the NNBI Team 2011 Proceedings of the 30th ICPIG, Belfast, Northern-Ireland

[19] Fantz U and Heger B 1998 Plasma Phys. Control. Fusion 402023

[20] Fantz U, Falter H, Franzen P, Wünderlich D, Berger M, Lorenz A, Kraus W, McNeely P, Riedl R and Speth E 2006 Nucl. Fusion 46 S297

[21] Álvarez R, Rodero A and Quintero M C 2002 Spectrochimica Acta Part B: Atomic Spectroscopy 571665 - 1680

[22] Ramsey A T and Diesso M 1999 Rev. Sci. Instr. 70 380-383

[23] Arancibia Monreal J, Chabert P and Godyak V 2013 Physics of Plasmas 20103504 
[24] Song S H, Yang Y, Chabert P and Kushner M J 2014 Physics of Plasmas 21093512

[25] Shimada M, Tynan G R and Cattolica R 2008 Journal of Applied Physics 103 033304 